\title{
ON THE EQUIVALENCE OF MULTIPLICITY AND THE GENERALIZED TOPOLOGICAL DEGREE
}

\author{
BY \\ T. O'NEIL AND J. W. THOMAS
}

\begin{abstract}
In this paper we first extend the definition of the multiplicity (as defined by $\mathrm{J}$. Cronin-Scanlon) of operators of the form $I+C+T$ to operators of the form $H+C+T$. We then show that the generalized topological degree (as defined by F. E. Browder and W. V. Petryshyn) of operators of the form $H+C+T$ is also defined. Finally, we show that when both the multiplicity and generalized topological degree of $H+C+T$ are defined, they are equal.
\end{abstract}

1. Introduction. In [3], [4], [5], [6] and [7] J. Cronin-Scanlon studied operators on a Banach space of the form $(I+C+T)(x)=y$, where $C$ is a compact linear operator and $T$ satisfies a contraction condition near the origin. The Leray-Schauder degree of $I+C+T$, in general, is not defined. Cronin-Scanlon's method for investigating the existence of solutions of the equation $(I+C+T)(x)=y$ was to define the multiplicity of $I+C+T$, denoted by $m(I+C+T)$, which had properties similar to the topological degree of an operator. Using these properties she was then able to examine such questions as existence of solutions and the number of solutions. In [5] she actually defined the degree of a mapping in the form $I+C+T$ (along with some additional conditions) defined on a Hilbert space in terms of $m(I+C+T)$.

In [1] and [2] F. E. Browder and W. V. Petryshyn extended the definition of topological degree to $A$-proper mappings. This class of mappings is much larger than the class of mappings for which the Leray-Schauder degree is defined. In this paper we shall show that the mappings of the form $I+C+T$ can be investigated through the generalized degree of Browder and Petryshyn. We shall show that not only is the generalized topological degree of $I+C+T$ defined with respect to some neighborhood of $\theta$, but it is, in fact, equal (up to a multiplicative \pm 1 ) to $m(I+C+T)$. Thus we see that the theory of generalized topological degree can then be applied to the integral equations that were studied by Schmidt [14].

In $\S 2$ instead of stating the results which we need from [3], we shall extend the definition of multiplicity of an operator to include mappings of the form $H+C+T$, where $H$ is a linear homeomorphism. In $\S 3$ we shall show that not only is the map $H+C+T$ (defined on a Banach space with a certain projectional structure) $A$ proper with respect to some neighborhood of $\theta$, but also that it satisfies a theorem

Presented to the Society, January 17, 1972; received by the editors July 29, 1970 and, in revised form, May 20, 1971.

AMS 1969 subject classifications. Primary 4780; Secondary 4770, 4530.

Key words and phrases. Topological degree, generalized topological degree, multiplicity of an operator, compact mappings, $A$-proper mappings.

Copyright $(1)$ 1972, American Mathematical Society 
of Browder and Petryshyn which will give the generalized degree of $H+C+T$ some especially nice properties. In $\$ 4$ we shall then show that if the Banach space on which $H+C+T$ is defined has the projectional structure that allows the generalized topological degree of $H+C+T$ to be defined, then this degree is, in fact, \pm 1 times the multiplicity of $H+C+T$. Finally in $\S 5$ we shall give an example to which the above work can be applied.

We would like to thank the referee of this paper for his many helpful comments and his very careful reading.

2. Multiplicity. Let $X$ be a Banach space, $H$ a linear homeomorphism mapping $X$ into $X$ and $C$ a compact linear map from $X$ into $X$. Let $X_{1}=N(H+C)=$ null space of $H+C$ and $X_{1}^{*}=N\left(H^{*}+C^{*}\right)$. In [13] F. Riesz obtained results for $N(I+C)$ where $C$ is compact. Analogously, and hence presented without proof, we obtain the following results: (1) $X_{1}$ is finite dimensional, (2) $X_{1}^{*}$ is finite dimensional and dimension $X_{1}=$ dimension $X_{1}^{*}$, and (3) there exists a projection from $X$ onto $X_{1}$, $E_{1}$, such that $X=X_{1}+X^{1}$ where $X^{1}=E^{1} X=\left(I-E_{1}\right) X$.

Now let $x_{1}, \ldots, x_{n}$ and $f_{1}, \ldots, f_{n}$ be bases for $X_{1}$ and $X_{1}^{*}$ respectively. Then there exist $g_{1}, \ldots, g_{n} \in X^{*}$ and $y_{1}, \ldots, y_{n} \in X$ such that

(1) for $x \in X, E_{1}(x)=\sum_{i=1}^{n} g_{i}(x) x_{i}\left(g_{i}\left(x_{j}\right)=\delta_{i j}\right)$, and

(2) $f_{i}\left(y_{j}\right)=\delta_{i j}$.

Denote by $C_{0}$ the transformation

$$
C x+\sum_{i=1}^{n} g_{i}(x) y_{i}
$$

$C_{0}$ is then compact and $N\left(H+C_{0}\right)=\{\theta\}$. Thus $H+C_{0}$ has a linear inverse $R=$ $H_{1}+C_{1}$ where $H_{1}$ is a linear homeomorphism $\left(H_{1}=H^{-1}\right)$ and $C_{1}$ is linear and compact. Then, analogous to the result of Cronin-Scanlon [7], we can show that, for all $x \in X, R(H+C)(x)=\left(I-E_{1}\right) x=E^{1} x$.

Along with the above facts we shall need the Implicit Function Theorem which is due to Hildebrandt and Graves [9].

Theorem 1 (Implicit Function Theorem). Let $X, Y, Z$ be Banach spaces and let $U, V, W$ be open sets in $X, Y, Z$, respectively. Let $L$ be a function with domain $U \times V \times W$ and range a subset of $X$. Assume that the following conditions are satisfied:

(1) there is a point $\left(x_{0}, y_{0}, z_{0}\right) \in U \times V \times W$ such that $x_{0}=L\left(x_{0}, y_{0}, z_{0}\right)$,

(2) there exists a positive number $c<1$ such that $\left\|L\left(x_{1}, y, z\right)-L\left(x_{2}, y, z\right)\right\| \leqq$ $c\left\|x_{1}-x_{2}\right\|$ for every $\left(x_{1}, y, z\right),\left(x_{2}, y, z\right) \in U \times V \times W$,

(3) $L$ is uniformly continuous on $U \times V \times W$.

Then the following conditions hold:

(1) for each $(y, z) \in V \times W$ there is at most one point $(x, y, z) \in U \times V \times W$ which satisfies the equation

$$
x=L(x, y, z)
$$


(2) there exist open sets $V_{1}$ and $W_{1}$, spherical neighborhoods of $y_{0}$ and $z_{0}$, respectively, and a function $F$ with domain $V_{1} \times W_{1}$ and range a subset of $X$ such that the point $(F(y, z), y, z)$ is an element of $U \times V \times W$ and $x=F(y, z)$ is a solution of equation (1) for every $(y, z) \in V_{1} \times W_{1}$,

(3) the solution $F(y, z)$ of equation (1) is uniformly continuous on $V_{1} \times W_{1}$.

We are now ready to consider the equation

$$
(H+C+T)(x)=y .
$$

We suppose that $T$ satisfies the following properties:

(1) $T$ is defined on some ball $B$ in $X$ centered at $\theta$,

(2) $T(\theta)=\theta$, and

(3) if $x, y \in B$, then $\|T x-T y\| \leqq M(x, y)\|x-y\|$ where $M(x, y)$ is a positive-valued function such that

$$
\lim _{(x, y) \rightarrow(\theta, \theta)} M(x, y)=0 .
$$

We note that when $(H+C)^{-1}$ exists, equation (2) can be multiplied by $(H+C)^{-1}$ and solved by the Implicit Function Theorem. If $H+C$ does not have an inverse, we multiply both sides of equation (2) by $E_{1} R$ and $E^{1} R$, and hence reduce equation (2) to the equations

$$
\begin{aligned}
E_{1} R T\left(x_{1}+x^{1}\right) & =E_{1} R y, \\
x^{1}+E^{1} R T\left(x_{1}+x^{1}\right) & =E^{1} R y,
\end{aligned}
$$

where $x_{1}=E_{1} x$ and $x^{1}=E^{1} x$.

If we then apply the Implicit Function Theorem to equation (4), we have the following theorem.

TheOREM 2 (CRONIN-SCANLON [3]). There exist open sets, $V_{1}$ and $W_{1}$, spherical neighborhoods of $x_{1}=\theta$ and $y=\theta$, respectively, and a uniformly continuous function $F$ with domain $V_{1} \times W_{1}$ and range in $X$, such that $x^{1}=F\left(x_{1}, y\right)$ is a solution to equation (4).

We then substitute $x^{1}=F\left(x_{1}, y\right)$ into equation (3) to obtain

$$
E_{1} R T\left(x_{1}+F\left(x_{1}, y\right)\right)-E_{1} R y=\theta .
$$

Solving equation (5) is obviously equivalent to solving equation (2). Thus we make the following definition.

DEFINITION 1. The multiplicity of solutions of the equation

$$
(H+C+T)(x)=y_{0},
$$

denoted by $m(H+C+T)$, is defined as

$$
\begin{aligned}
m(H+C+T) & =+1, \quad \text { when }(H+C)^{-1} \text { exists, } \\
& =d\left(E_{1} R T\left(x_{1}+F\left(x_{1}, y_{0}\right)\right)-E_{1} R\left(y_{0}\right)_{X_{1}}, B \cap X_{1}, \theta\right), \text { otherwise, }
\end{aligned}
$$


where $B$ is a sufficiently small ball in $X$ centered at $\theta$ and the degree above is the usual Brouwer degree.

Cronin-Scanlon, in [3], using the definition of multiplicity similar to Definition 1, places an additional condition on $T$ and then derives methods for calculating $m(I+C+T)$. These methods would also be applicable to calculating $m(H+C+T)$.

3. The generalized degree of $H+C+T$. In [1] and [2], F. E. Browder and W. V. Petryshyn defined a generalized topological degree. We shall be interested in a slight variation of some of their definitions and results. Definitions 2-4 below are the same as those given in [2] except that we shall replace their mapping $P_{n}$, which maps $X_{n}$ into $X$, by the inclusion map. The inclusion map will surely satisfy the properties of the mapping $P_{n}$. We then obtain the following definitions.

Definition 2 [2, Definition 3.1]. Let $X$ be a real Banach space. By a projective approximation scheme for mappings from $X$ into $X$, denoted by $\Gamma$, we mean a sequence of oriented finite-dimensional subspaces $\left\{X_{n}\right\}$ and a sequence of linear projections from $X$ onto $X_{n},\left\{Q_{n}\right\}$, such that $Q_{n} x \rightarrow x$ for each $x \in X$.

It can be shown [2] under the conditions of Definition 2 that we also get (1) $\left\{Q_{n}\right\}$ is uniformly bounded and (2) if $F$ is a given finite-dimensional subspace of $X$, then there exists an integer $n_{F} \geqq 1$ such that $Q_{n}$ is injective on $F$ for each $n \geqq n_{F}$.

Definition 3 [2, Definition 1.2]. Let $G$ be an open subset of $X$ and $W$ a mapping of $\bar{G}$ (the closure of $G$ ) into $X . W$ is said to be A-proper on $\bar{G}$ with respect to a given approximation scheme $\Gamma=\left(\left\{X_{n}\right\},\left\{Q_{n}\right\}\right)$ if and only if, for any sequence $\left\{n_{j}\right\}$ of positive integers with $n_{j} \rightarrow \infty$ and a corresponding sequence $\left\{x_{n_{j}} \mid x_{n_{j}} \in X_{n_{j}}\right\}$ with $x_{n_{j}} \in \bar{G}$ such that $\left\|Q_{n_{j}} W x_{n_{n}}-Q_{n_{j}} y\right\| \rightarrow 0$ for some $y \in X$, there exists an infinite subsequence $\left\{x_{n_{j(k)}}\right\}$ and an element $x \in X$ such that $x_{n_{j(k)}} \rightarrow x$ and $W x=y$.

Let us next introduce the notation $W_{n}=Q_{n} W$ and proceed to define the generalized topological degree as follows.

Definition 4 [2, Definition 1.3]. Let $W$ be an A-proper continuous mapping from $\bar{G}$ into $X$ with respect to $\Gamma$. Let $G_{n}=X_{n} \cap G$ be bounded for all $n$ and suppose that $a \notin W(\partial G)$.

We define $D(W, G, a)$, the degree of $W$ on $G$ over a with respect to $\Gamma$, as follows: Let $Z^{\prime}$ be the set of all integers together with $\pm \infty$. Then $D(W, G, a)$ is the subset of $Z^{\prime}$ given by $D(W, G, a)=\left\{\gamma \mid \gamma \in Z^{\prime}\right.$, there exists an infinite sequence $\left\{n_{j}\right\}$ of positive integers with $n_{j} \rightarrow \infty$ such that $\left.d\left(W_{n_{j}}, G_{n_{j}}, Q_{n_{j}} a\right) \rightarrow \gamma\right\}$.

To keep the concepts of generalized degree separate from those of the more classical degree, we shall use a capital $D$ to denote the generalized degree and a lower case $d$ to denote the Brouwer degree (when in $R^{n}$ ) and the Leray-Schauder degree.

The results we obtain in this paper do not use the most general form of the generalized degree as defined above. Whenever we are confronted with the generalized degree of a function we shall be able to apply the following theorem. This theorem is Theorem 2 in [2] and for that reason we do not include the proof. 
THEOREM 3. Let $h$ and $K$ be two continuous mappings of $\bar{G}$ into $X$ such that if we assume $G_{n}=X_{n} \cap G$ are bounded and set $h_{n}=\left.Q_{n} h\right|_{\bar{G}_{n}}$, the following hypotheses are satisfied:

(1) $h$ is an A-proper homeomorphism of $G$ onto an open subset $h(G)$ of $X$ with $h$ mapping $\bar{G}$ homeomorphically onto $\overline{h(G)}$.

(2) For each $n, h_{n}$ is a homeomorphism of $G_{n}$ onto the open subset $h_{n}\left(G_{n}\right)$ of $X_{n}$ and $h_{n}$ maps $\bar{G}_{n}$ homeomorphically onto $\overline{h(G)}$.

(3) There exists a continuous function $\alpha:[0, \infty) \rightarrow[0, \infty)$ such that $r_{i} \rightarrow 0$ whenever $\alpha\left(r_{i}\right) \rightarrow 0$, for which $\left\|h_{n} u-h_{n} v\right\| \geqq \alpha(\|u-v\|)$ for all $u$ and $v$ in $G_{n}$ and all $n \geqq 1$.

(4) $K$ is compact.

Consider now the mapping $W=h+K: \bar{G} \rightarrow X$ and suppose that $a \notin W(\partial G)$. Then the following are true:

(1) $W$ is A-proper.

(2) There exists an integer $n_{0} \geqq 1$ such that $d\left(W_{n}, G_{n}, Q_{n} a\right)$ is defined for each $n \geqq n_{0}$.

(3) There exists an integer $n_{1} \geqq 1$ such that, for all $n \geqq n_{1},\left|d\left(W_{n}, G_{n}, Q_{n} a\right)\right|$ $=\left|d\left(I+K h^{-1}, h(G), a\right)\right|$ and is independent of the choice of $n$.

(4) $D(W, G, a)=\left\{ \pm d\left(I+K h^{-1}, h(G), a\right)\right\}$.

In [2], condition (2) required that $h_{n}$ be an orientation preserving homeomorphism. If this condition is assumed, then result (3) becomes

$$
d\left(W_{n}, G_{n}, Q_{n} a\right)=d\left(I+K h^{-1}, h(G), a\right)
$$

and condition (4) becomes

$$
D(W, G, a)=\left\{d\left(I+K h^{-1}, h(G), a\right)\right\} .
$$

Since our result will be unchanged by a multiplicative \pm 1 , it is not necessary to assume that $h_{n}$ be orientation preserving. For convenience, however, we shall assume that $h_{n}$ is orientation preserving in our lemmas and theorems. This will eliminate many cumbersome \pm 1 's.

In this paper we wish to apply the generalized topological degree to the mapping $H+C+T$. This mapping is not, in general, $A$-proper with respect to any open set $G$. For this reason we say that a map $f$ is locally A-proper at $\theta$ if there exists an open neighborhood of $\theta$, say $N$, such that $f$ is $A$-proper with respect to $N$. We are then able to prove the following lemma.

Lemma $1 . I+T$ is locally A-proper at $\theta$.

Proof. Let $K_{1}$ be the uniform bound on $\left\|Q_{n}\right\|$. Using condition(3) in the definition of $T$ we choose $r$ so that $M(x, y) \leqq k<\operatorname{minimum}\left\{\frac{1}{2}, 1 / K_{1}, K_{1}\right\}$ in $B_{r} \times B_{r}$ and $r_{2}=r / 4 K_{1}$. We shall prove that $I+T$ is $A$-proper on $B_{r_{2}}$.

Let $G=B_{r_{2}}$ and denote $I+T$ by $W$. Let $\left\{n_{j}\right\}$ be a subsequence of the integers, $x_{n_{j}} \in \bar{G}_{n_{j}}$ and suppose there exists a $y \in X$ such that $\left\|W_{n_{j}} x_{n_{j}}-Q_{n_{j}} y\right\| \rightarrow 0$. 
We note that since

$$
\left\|W_{n,} x_{n,}-y\right\| \leqq\left\|W_{n_{j}} x_{n_{j}}-Q_{n_{j}} y\right\|+\left\|Q_{n,} y-y\right\|,
$$

the above assumption and Definition 2 imply that $\left\|W_{n_{j}} x_{n_{j}}-y\right\| \rightarrow 0$. We also note that since

$$
\left\|W_{n_{j}} x_{n_{j}}\right\|=\left\|Q_{n_{j}}(I+T) x_{n_{j}}\right\| \leqq K_{1}(1+k)\left\|x_{n_{j}}\right\|<2 K_{1} r_{2}=r_{1},
$$

then $y \in B_{r_{1}}$ where $r_{1}=2 K_{1} r_{2}$.

Claim. There exists a $z \in \bar{B}_{r}$ such that $W z=y$.

Define $f_{y}$ by $f_{y}(x)=y-T x$, let $t_{0} \in B_{r_{2}}$ and define $t_{n+1}=f_{y}\left(t_{n}\right)$. In an approach similar to that used in the proof of the Banach Fixed Point Theorem we can show that $t_{n} \in B_{r}$ for all $n$ and $\left\{t_{n}\right\}$ is a Cauchy sequence. Let $t_{n} \rightarrow z$. Then $W z=y$. We then have that

$$
\begin{aligned}
\left\|W_{n_{j}} x_{n_{j}}-y\right\| & =\left\|Q_{n_{j}} W x_{n_{j}}-W z\right\|=\left\|\left(x_{n_{j}}-z\right)-\left(T z-Q_{n_{j}} T x_{n_{j}}\right)\right\| \\
& \geqq\left\|x_{n_{j}}-z\right\|-\left\|\left(T z-Q_{n_{j}} T z\right)+\left(Q_{n_{j}} T z-Q_{n_{j}} T x_{n_{j}}\right)\right\| \\
& \geqq\left\|x_{n_{j}}-z\right\|-\left\|Q_{n_{j}} T z-Q_{n_{j}} T x_{n_{j}}\right\|-\left\|T z-Q_{n_{j}} T z\right\| \\
& \geqq\left\|x_{n_{j}}-z\right\|\left(1-K_{1} k\right)-\left\|T z-Q_{n_{j}} T z\right\| .
\end{aligned}
$$

Since $\left\|W_{n_{j}} x_{n_{j}}-y\right\| \rightarrow 0,\left\|T z-Q_{n_{j}} T z\right\| \rightarrow 0$ and $\left(1-K_{1} k\right)>0$, we have that $x_{n_{j}} \rightarrow z$.

We now use Lemma 1 to prove that $H+T$ is also locally $A$-proper at $\theta$.

THEOREM 4. $H+T$ is locally A-proper at $\theta$.

Proof. We shall show that $H+T$ is $A$-proper on some ball, $B_{r}=G$, such that $B_{r} \subset H^{-1}\left(B_{r_{2}}\right)$, where $r_{2}$ is as in Lemma 1 .

Let $\left\{n_{j}\right\}$ be a subsequence of integers. Suppose that $x_{n_{j}} \in \bar{G}_{n_{j}}$ and that there exists a $y \in X$ such that $\left\|Q_{n_{j}}(H+T) x_{n_{j}}-Q_{n,} y\right\| \rightarrow 0$. But this is the same as

$$
\left\|Q_{n_{j}}\left(I+T H^{-1}\right)\left(H x_{n_{j}}\right)-Q_{n_{j}} y\right\| \rightarrow 0 .
$$

Furthermore, it is easy to show that $T H^{-1}$ will also satisfy the conditions of Lemma 1 (with $\left\|H^{-1}\right\| M(x, y)$ replacing $M(x, y)$ in condition (3) on $T$ ). Therefore by Lemma $1, H x_{n_{j}} \rightarrow z$ and $\left(I+T H^{-1}\right) z=y$. But then $x_{n_{j}} \rightarrow H^{-1}(z)$ and

$$
(H+T)\left(H^{-1} z\right)=y .
$$

Thus $H+T$ is locally $A$-proper at $\theta$.

Thus we have that $H+T$ is $A$-proper on some $B_{r}$. It is not hard, then, to show that $(H+T)+C$ is $A$-proper on $B_{r}$. However, we are able to get more than $(H+T)+C$ being $A$-proper. We shall, in the following lemmas, show that $(H+T)+C$ is such that it will satisfy Theorem 3 .

LEMMA 2. $h=H+T$ is a homeomorphism from some ball $B_{r}$ onto $h\left(B_{r}\right) . h\left(B_{r}\right)$ is open and $h(\bar{U})=\overline{h(U)}$ for any open set $U$ such that $\bar{U} \subset B_{r}$. 
Proof. The fact that $h$ is a homeomorphism follows from

$$
\begin{aligned}
\|x-y\|[m-k] & \leqq\|H x-H y\|-\|T y-T x\| \leqq\|h x-h y\| \\
& \leqq\|H x-H y\|+\|T x-T y\| \leqq(\|H\|+k)\|x-y\|
\end{aligned}
$$

where $m=\left\|H^{-1}\right\|^{-1}$ and $B_{r}$ is chosen so that $M(x, y)<k$ for $x, y \in B_{r}$ and $k<m$. The proof that $h\left(B_{r}\right)$ is open and $h(\bar{U})=\overline{h(U)}$ is similar to that for the case when $H=I$ and $T$ is a strict contraction.

LEMMA 3. $h_{n}$ is a homeomorphism from some ball $X_{n} \cap B_{r}$ onto $h_{n}\left(X_{n} \cap B_{r}\right)$. $h_{n}\left(X_{n} \cap B_{r}\right)$ is open and $h_{n}(\bar{U})=\overline{h(U)}$ for any open set $U$ such that $\bar{U} \subset B_{r}\left(h_{n}=Q_{n} h\right)$.

Proof. We let $k_{n}=I_{n}+Q_{n} T H^{-1}$ where $I_{n}$ is the identity map on $X_{n}$. Then since

$$
\left(1-k K_{1}\left\|H^{-1}\right\|\right)\|x-y\| \leqq\left\|k_{n} x-k_{n} y\right\| \leqq\left(1+k K_{1}\left\|H^{-1}\right\|\right)\|x-y\|,
$$

we see that if we choose $r$ such that $m(x, y)<k<1 / K_{1}\left\|H^{-1}\right\|$ for $x, y \in B_{r}$, then $k_{n}$ is a homeomorphism on $B_{r}$. Then $h_{n}=k_{n} H$ will also be a homeomorphism. The last part of the lemma is similar to the corresponding part of Lemma 2.

LEMMA 4. $h_{n}$ satisfies condition (3) of Theorem 3.

Proof. We note that

$$
\begin{aligned}
\left\|h_{n} u-h_{n} v\right\|=\left\|k_{n} H u-k_{n} H v\right\| & \geqq\|H u-H v\|\left(1-k K_{1}\left\|H^{-1}\right\|\right) \\
& \geqq \frac{1}{\left\|H^{-1}\right\|}\|u-v\|\left(1-k K_{1}\left\|H^{-1}\right\|\right)
\end{aligned}
$$

where $k_{n}$ is as in Lemma 3. Thus we let

$$
\alpha(r)=\left\|H^{-1}\right\|^{-1}\left(1-k K_{1}\left\|H^{-1}\right\|\right) r
$$

and condition (3) of Theorem 3 is satisfied.

Since $C$ is compact, the mapping $H+C+T$ satisfies the hypotheses of Theorem 3 . We then have the following theorem.

THEOREM 5. There exists an $r$ such that $D\left(H+C+T, B_{r}, \theta\right)$ is defined and such that $D\left(H+C+T, B_{r}, \theta\right)=\left\{d\left(I+C h^{-1}, h\left(B_{r}\right), \theta\right)\right\}$ where $h=H+T$.

We can now use the properties of generalized degree to investigate the existence of solutions of $(H+C+T)(x)=\theta$. In [5], Cronin-Scanlon proved the invariance under homotopy of the special degree defined in that paper. We see that since $D\left(H+C+T, B_{r}, \theta\right)$ is defined for some $r$, the invariance under homotopy follows from that corresponding property in the paper by Browder and Petryshyn [2].

We should note that the definition of multiplicity was for the equation

$$
(H+C+T)(x)=y
$$

for $y$ in a sufficiently small neighborhood of $\theta$. We can, however, use the property 
that if $D\left(H+C+T, B_{r}, \theta\right)$ is defined then there exists a neighborhood of $\theta, N$, such that for any $y \in N, D\left(H+C+T, B_{r}, y\right)$ is defined and is equal to

$$
D\left(H+C+T, B_{r}, \theta\right) \text {. }
$$

4. The equality of the generalized degree and multiplicity. In this section we shall show that the multiplicity is, in fact, a special case of the generalized degree. We shall show that

$$
|m(H+C+T)|=\left|d\left(I+C h^{-1}, h\left(B_{r}\right), \theta\right)\right| .
$$

When $H+C$ is nonsingular, then $m(H+C+T)=1$. It is not hard to show that when $H+C$ is injective so also is $H+C+T$. It is then clear that $d\left(I+C h^{-1}, h(G), \theta\right)$ $= \pm 1$ and that $(6)$ is satisfied.

When $H+C$ is singular, we proceed much as Cronin-Scanlon did in [3]. We define $\Phi$ and $\Psi$ as follows:

$$
\begin{aligned}
& \Phi(x)=x+E^{1} R T(x), \\
& \Psi(x)=E^{1} x+E_{1} R T\left[E_{1}(x)+F\left(E_{1} x, E^{1} x\right)\right],
\end{aligned}
$$

where $R=\left(H+C_{0}\right)^{-1}=H_{1}+C_{1}$ and $F$ is as in (5). We note that

$$
R(H+C+T)=R(h+C)=\Psi \Phi .
$$

This can be rewritten in the form

$$
\left(I+C_{1} H_{1}^{-1}\right) H_{1}\left(I+C h^{-1}\right)=\Psi \Phi h^{-1} .
$$

Before stating Theorem 7 we state the product theorem which can be found in [11].

THEOREM 6. Let $f=I+C_{1}$ and $g=I+C_{2}$ be two mappings of subsets of the Banach space $X$ into $X$ such that

(1) $C_{1}$ and $C_{2}$ are compact,

(2) $G$ is a bounded open set such that $C_{1}$ is defined on $\bar{G}$ and $C_{2}$ is defined on the closure of $A$, a bounded open set containing $\left(I+C_{1}\right)(\bar{G})$,

(3) $p \notin g f(\partial G) \cup g[\partial f(G)]$.

Then

$$
d(g f, G, p)=\sum_{(i)} d\left(f, g, d_{i}\right) d\left(g, D_{i}, p\right)
$$

where $\sum_{(i)}$ means that the sum over the bounded components of $X-f(\partial G), D_{i}$, and $d_{i}$ is an arbitrary element of $D_{i}$. Only a finite number of the terms on the right will be nonzero.

Using the above product theorem we prove a slightly more general product theorem that will help us investigate equation (8).

THEOREM 7. Let $f=H+C_{1}$ and $g=I+C_{2}$ be two mappings of subsets of the Banach space $X$ into $X$ such that 
(1) $H$ is a homeomorphism which satisfies the hypotheses of Theorem 3 and $C_{1}$ and $C_{2}$ are compact.

(2) $G$ is a bounded open set such that $H$ and $C_{1}$ are defined on $\bar{G}$ and $C_{2}$ is defined on $D$ where $D$ is a bounded open set containing $f(\bar{G})$.

(3) $p \notin g f(\partial G) \cup g[\partial f(G)]$.

Then

$$
D(g f, G, p)=\sum_{(i)} d\left(g, D_{i}, p\right) D\left(f, G, d_{i}\right)
$$

where the sum is taken over all of the components of $X-f(\partial G), D_{i}$, and $d_{i}$ is an arbitrary element of $D_{i}$. Only a finite number of the terms on the right will be nonzero.

It should be remarked that since $f$ is such that each of the sets $D\left(f, G, d_{i}\right)$ is the singleton set, by $\sum_{(i)} d\left(g, D_{i}, p\right) D\left(f, G, d_{i}\right)$ we merely mean the sum and product of the integers involved.

Proof. Since $H$ is such that it satisfies the hypotheses of Theorem 3, then $g f=\left(I+C_{2}\right)\left(H+C_{1}\right)=H+C_{1}+C_{2}\left(H+C_{1}\right)$ also satisfies Theorem 3 and

$$
D(g f, G, p)=\left\{d\left(I+C_{1} H^{-1}+C_{2}\left(H+C_{1}\right) H^{-1}, H(G), p\right)\right\} .
$$

Since $I+C_{1} H^{-1}+C_{2}\left(H+C_{1}\right) H^{-1}=\left(I+C_{2}\right)\left(I+C_{1} H^{-1}\right)=g f_{1},\left(f_{1}=I+C_{1} H^{-1}\right)$ we see that

$$
\begin{aligned}
d\left(I+C_{1} H^{-1}+C_{2}(H+\right. & \left.\left.C_{1}\right) H^{-1}, H(G), p\right) \\
& =d\left(\left(I+C_{2}\right)\left(I+C_{1} H^{-1}, H(G), p\right)=d\left(g f_{1}, H(G), p\right) .\right.
\end{aligned}
$$

We now use Theorem 6 to get

$$
d\left(g f_{1}, H(G), p\right)=\sum_{(i)} d\left(I+C_{1} H^{-1}, H(G), e_{i}\right) d\left(I+C_{2}, E_{i}, p\right),
$$

where $E_{i}$ is a component of $X-f_{1}(\partial H(G))$ and $e_{i} \in E_{i}$.

It is not hard to show that $p \notin g[f(\partial G)] \cup g[\partial f(G)]$ implies that

$$
p \notin g\left[f_{1}(\partial H(G))\right] \cup g\left[\partial f_{1}(H(G))\right]
$$

(so that Theorem 6 can be applied).

Since $f_{1}(H(G))$ clearly equals $f(G)$, we see that $E_{i}=D_{i}$ and $e_{i}=d_{i}$.

Therefore, since

$$
D\left(f, G, d_{i}\right)=D\left(H+C_{1}, G, d_{i}\right)=\left\{d\left(I+C_{1} H^{-1}, H(G), d_{i}\right)\right\},
$$

we see that

$$
D(g f, G, p)=\sum_{(i)} d\left(g, D_{\imath}, p\right) D\left(f, G, d_{i}\right) .
$$

COROLlaRY 1. If $f(\theta)=\theta$ is an isolated $\theta$-point of $g$, then there exists an $r$ such that, when $G=B_{r}$,

for only one $i$.

$$
d\left(g, D_{i}, p\right) D\left(f, G, d_{i}\right) \neq\{0\}
$$


Corollary 1 follows from Theorem 7 in much the same way as Lemma 5.2 follows from Lemma 5.1 in [3].

We can now apply Corollary 1 to equation (8) and obtain

(9) $d\left(I+C_{1} H_{1}^{-1}, D_{i}, \theta\right) D\left(H_{1}\left(I+C h^{-1}\right), G, d_{i}\right)=d\left(\Psi, D_{i}, \theta\right) D\left(\Phi h^{-1}, G, d_{i}\right)$.

We can then make use of the following lemmas.

Lemma 5. $d\left(I+C_{1} H_{1}^{-1}, D_{i}, \theta\right)= \pm 1$.

Proof. Once we note that $I+C_{1} H_{1}^{-1}$ is injective, the lemma follows from LeraySchauder [10, pp. 56-59].

LEMMA 6. $\Phi h^{-1}$ is injective on some neighborhood of $\theta$ and satisfies the hypotheses of Theorem 3.

Proof. Suppose that $\Phi\left(h^{-1} x\right)=\Phi\left(h^{-1} y\right)$, i.e.

$$
h^{-1} x+E^{1} R T^{-1} x=h^{-1} y+E^{1} R T h^{-1} y \text {. }
$$

But then we also have $x+h E^{1} R T h^{-1} x=y+h E^{1} R T h^{-1} y$. We note that

$$
\begin{aligned}
\left\|\left(x+h E^{1} R T h^{-1} x\right)-\left(y+h E^{1} R T h^{-1} y\right)\right\| & \geqq\|x-y\|-\left\|h E^{1} R T h^{-1} y-h E^{1} R T h^{-1} x\right\| \\
& \geqq\|x-y\|\left[1-\left\|h E^{1} R\right\| M\left(h^{-1} y, h^{-1} x\right)\left\|h^{-1}\right\|\right] .
\end{aligned}
$$

Choose $r$ small enough so that $x, y \in B_{r}$ implies that

$$
1-\left\|h E^{1} R\right\|\left\|h^{-1}\right\| M\left(h^{-1} y, h^{-1} x\right)>0 .
$$

Then if $x \neq y$, we have a contradiction.

$\Phi h^{-1}$ satisfies the hypotheses of Theorem 3 since it is very similar to the mapping $H+T+C$ (with $C \equiv 0$ ).

Lemma 7. $D\left(\Phi h^{-1}, G, d_{i}\right)=\{ \pm 1\}(+1$ or -1 but not both $)$.

Proof. Using the fact that in $G, \Phi h^{-1}$ is injective, this lemma follows in the same manner as Lemma 5.

Lemma 8. There exists a $B_{r}$ such that $d\left(\Psi, D_{i}, \theta\right)=d\left(\Psi, B_{r}, \theta\right)$.

Proof. Note that $\Psi(\theta)=\theta$ and $\theta \in D_{i}$. Choose $B_{r}$ such that $B_{r} \subset D_{i}$. Then since $\theta$ is an isolated $\theta$-point of $\Psi$, we get the above result.

The following lemma is due to Cronin-Scanlon [3, Lemma 5.4].

LeMma 9. $d\left(\Psi, B_{r}, \theta\right)=d\left(\left.\Psi\right|_{X_{1}}, X_{1} \cap B_{r}, \theta\right)$.

The above lemmas and equation (9) then yield

$$
D\left(H_{1}\left(I+C h^{-1}\right), G, d_{i}\right)=\{ \pm m(H+C+T)\} .
$$

It then remains to show that

$$
D\left(H_{1}\left(I+C h^{-1}\right), G, d_{i}\right)= \pm D(H+C+T, G, \theta) .
$$


We should note that since $\theta \in D_{i}$, we can choose $d_{i}=\theta$.

To prove (11) we denote $C h^{-1}$ by $K$ and proceed with the following. Suppose that $\left\{y_{1}, \ldots, y_{m}\right\}$ is an $\varepsilon / 2$-net of $\overline{K(G)}$ (exists since $\overline{K(G)}$ is compact). Since, for any $x \in X, Q_{n}(x) \rightarrow x$, we see that for any $\varepsilon / 2>0$ there exists an $N_{i}$ such that $n \geqq N_{i}$ implies that $\left\|Q_{n}\left(y_{i}\right)-y_{i}\right\|<\varepsilon / 2$. Let $N=\max \left\{N_{1}, \ldots, N_{m}\right\}$ and consider $X_{n}$ where $n \geqq N$. Then for any $y \in \overline{K(G)}$ there exists a $y_{i}$ such that $\left\|y-y_{i}\right\|<\varepsilon / 2$. We then have that

$$
\left\|Q_{n}\left(y_{i}\right)-y\right\| \leqq\left\|Q_{n}\left(y_{i}\right)-y_{i}\right\|+\left\|y_{\imath}-y\right\|<\varepsilon .
$$

Thus $\left\{Q_{n}\left(y_{1}\right), \ldots, Q_{n}\left(y_{m}\right)\right\}$ is an $\varepsilon$-net of $\overline{K(G)}$.

We next define $F_{\varepsilon}$ by

where

$$
F_{\varepsilon}(x)=\sum_{i=1}^{m} m_{i}(x) Q_{n}\left(y_{i}\right) / \sum_{i=1}^{m} m_{i}(x)
$$

$$
\begin{aligned}
m_{i}(x) & =\varepsilon-\left\|x-Q_{n}\left(y_{i}\right)\right\| & & \text { if }\left\|x-Q_{n}\left(y_{i}\right)\right\| \leqq \varepsilon, \\
& =0 & & \text { if }\left\|x-Q_{n}\left(y_{i}\right)\right\|>\varepsilon,
\end{aligned}
$$

and choose $\varepsilon$ sufficiently small so that

$$
d\left(I+F_{\varepsilon} K, X_{n} \cap G, \theta\right)=d(I+K, G, \theta) .
$$

Let us now choose $N_{1}$ such that, for $n \geqq N_{1}$,

$$
d\left(Q_{n} H_{1}(I+K), G \cap X_{n}, \theta\right)=d\left(I+H_{1} K H_{1}^{-1}, H_{1}(G), \theta\right),
$$

and suppose from this point on that $n$ is always at least as large as $N$ and $N_{1}$. Now consider the following lemmas.

Lemma 10 [2, Proposition 1].

$$
d\left(Q_{n} H_{1}(I+K), G \cap X_{n}, \theta\right)=d\left(Q_{n} H_{1}\left(I+F_{\varepsilon} K\right), G \cap X_{n}, \theta\right) .
$$

Lemma 11. For $n$ sufficiently large we have $d\left(Q_{n} H_{1}, D_{i}, \theta\right)=d\left(I, H_{1}\left(D_{i}\right), \theta\right)=1$ if $\theta \in H_{1}\left(D_{i}\right)$.

Proof. This follows from Theorem 3.

We now state the following theorem which can be found in [8].

THEOREM 8. Suppose (1) the mapping $f$ is continuous in the closure of a bounded open set $\Omega \subset R^{n}$, (2) the mapping $g$ is continuous on $f(\bar{\Omega})$, and (3) $g(y) \neq u_{0}$ for $y \in f(\partial \Omega)$, where $u_{0}$ is fixed in $R^{n}$. Then

$$
d\left(g f, \Omega, u_{0}\right)=\sum_{(i)} d\left(f, \Omega, d_{\imath}\right) d\left(g, D_{i}, u_{0}\right)
$$

where we sum over all of the components of $R^{n}-f(\partial \Omega), D_{i}$, that are contained in $f(\Omega)$ and where $d_{i}$ is an arbitrary point in $D_{i}$.

Theorem 8 and the preceding lemmas allow us to prove the following product theorem. 
THEOREM 9. Let $H_{1}$ and $f=I+K$ be two mappings of subsets of the Banach space $X$ into $X$ such that

(1) $H_{1}$ is a homeomorphism which satisfies the hypotheses of Theorem 3 and $K$ is compact.

(2) $G$ is a bounded open subset of $X$ such that $K$ is defined on $\bar{G}$ and $H_{1}$ is defined on the closure $A$ of some bounded open set containing $f(\bar{G})$.

(3) $p \notin f(\partial G)$.

Then

$$
D\left(H_{1} f, G, p\right)=\sum_{(i)} d\left(f, G, d_{i}\right) D\left(H_{1}, D_{i}, p\right)
$$

where the sum is taken over all of the components, $D_{i}$, of $X-f(\partial G)$ that are contained in $f(\bar{G})$ and $d_{i}$ is an arbitrary point in $D_{i}$.

Proof. For sufficiently large $n$, Lemma 10 and Theorem 8 allow us to write

$$
\begin{aligned}
d\left(Q_{n} H_{1} f, X_{n} \cap G, p\right) & =d\left(Q_{n} H_{1}\left(I+F_{\varepsilon} K\right), X_{n} \cap G, p\right) \\
& =\sum_{(i)} d\left(Q_{n} H_{1}, X_{n} \cap D_{i}, p\right) d\left(I+F_{\varepsilon} K, X_{n} \cap G, d_{i}\right) .
\end{aligned}
$$

Our choice of $\varepsilon$ allows us to state that $d\left(I+F_{\varepsilon} K, X_{n} \cap G, d_{i}\right)=d\left(I+K, G, d_{i}\right)$ and our choice of $n$ allows us to write $\left\{d\left(Q_{n} H_{1}, X_{n} \cap D_{i}, p\right)\right\}=D\left(H_{1}, D_{i}, p\right)$. Thus we have the desired result.

We then have the following corollary.

Corollary 2. If $f(\theta)=\theta$ and $\theta$ is an isolated $\theta$-point of $H_{1}$, then

$$
D\left(H_{1} f, G, \theta\right)=d(f, G, \theta) D\left(H_{1}, D_{i}, \theta\right)
$$

for one component of $X-f(\partial G), D_{i}$.

With the above corollary and Lemma 11 we have

$$
D\left(H_{1}(I+K), G, \theta\right)=D\left(H_{1}\left(I+C h^{-1}\right), G, \theta\right)=\left\{d\left(I+C h^{-1}, G, \theta\right)\right\} .
$$

This result along with equation (10) yields

$$
D\left(H+C+T, B_{r}, \theta\right)=\{ \pm m(H+C+T)\} .
$$

Thus the value obtained by using the generalized degree and the multiplicity are the same up to a multiplicative constant \pm 1 .

5. An example. In this section we shall merely restate the example due to Cronin-Scanlon in [3]. Consider $X=C[0,1]$ (the continuous functions in $[0,1]$ ) with the sup-norm. It is shown in [12] that if we let

$$
\begin{aligned}
x_{0}(t) & =\chi_{[0,1]}(t) \quad(\text { the characteristic function of the set }[0,1]), \\
x_{1}(t) & =t \chi_{[0,1]}(t), \\
x_{2}(t) & =x_{1}(2 t)+\chi_{[0,1]}(2 t-1)-x_{1}(2 t-1), \\
x_{2^{n}+i}(t) & =x_{2}\left(2^{n} t-i+1\right), \quad i=1, \ldots, \quad n=1,2, \ldots
\end{aligned}
$$


then $\left\{x_{i}\right\}$ is a basis for $C[0,1]$. Since $\left\{x_{i}\right\}$ is a basis for $C[0,1]$, each $x \in C[0,1]$ can be expressed uniquely as $x=\sum_{i=0}^{\infty} a_{i} x_{i}$ (where the $a_{i}$ are real constants).

Let $X_{n}=\operatorname{span}\left\{x_{0}, \ldots, x_{n}\right\}$ and $Q_{n}(x)=Q_{n}\left(\sum_{i=0}^{\infty} a_{i} x_{i}\right)=\sum_{i=0}^{n} a_{i} x_{i}$. This sequence of subspaces and projections will be a projective approximation scheme for mappings from $C[0,1]$ into $C[0,1]$.

We then define $C$ by $C(x(t))=y(t)$ where $y(t)=x(1) \cdot t$ and define $T$ by $T(x(t))$ $=y(t)$ where $y(t)=[x(t)]^{2}$. Then $I+C+T$ will satisfy the conditions set forth in this paper. This is then an example of a mapping for which the generalized degree is defined on some ball about the origin (and hence the multiplicity) while neither the Leray-Schauder degree nor the extended degree due to Cronin-Scanlon [5] is defined.

\section{REFERENCES}

1. F. E. Browder and W. V. Petryshyn, The topological degree and Galerkin approximation for noncompact operators in Banach spaces, Bull. Amer. Math. Soc. 74 (1968), 641-646. MR 37 \#4678.

2. - Approximation methods and the generalized topological degree for nonlinear mappings in Banach spaces, J. Functional Analysis 3 (1969), 217-245. MR 39 \#6126.

3. J. Cronin-Scanlon, Branch points of solutions of equations in Banach spaces, Trans. Amer. Math. Soc. 69 (1950), 208-231. MR 12, 716.

4. - The existence of multiple solutions of elliptic differential equations, Trans. Amer. Math. Soc. 68 (1950), 105-131. MR 11, 361.

5. - A definition of degree for certain mappings in Hilbert space, Amer. J. Math. 73 (1951), 763-772. MR 13, 662.

6. - Branch points of solutions of equations in Banach space. II, Trans. Amer. Math. Soc. 76 (1954), 207-222. MR 16, 47.

7. - Fixed points and topological degree in nonlinear analysis, Math. Surveys, no. 11, Amer. Math. Soc., Providence, R. I., 1964. MR 29 \#1400.

8. E. Heinz, An elementary analytic theory of the degree of mapping in n-dimensional space, J. Math. Mech. 8 (1959), 231-247. MR 21 \#1370.

9. T. H. Hildebrandt and L. M. Graves, Implicit functions and their differentials in general analysis, Trans. Amer. Math. Soc. 29 (1927), 127-153.

10. J. Leray and J. Schauder, Topologie et équations fonctionelles, Ann. Sci. École Norm. Sup. (3) 51 (1934), 45-78.

11. J. Leray, Topologie des espaces abstraits de M. Banach, C. R. Acad. Sci. Paris 200 (1935), 1082-1084 (note).

12. Jurg T. Marti, Introduction to the theory of bases, Springer-Verlag, New York, 1969.

13. F. Riesz, Uber lineare Funktionalgleichungen, Acta Math. 41 (1918), 71-98.

14. E. Schmidt, Zur Theorie der linearen und nichtlinearen Integralgleichungen, Math. Ann. 65 (1907/08), 370-399.

Department of Mathematics, University of Wyoming, LaRamie, Wyoming 82070 